\title{
Diagnosis of motor neuron disease by neurologists: a study in three countries
}

\author{
Ting-Ming Li, Michael Swash, Eva Alberman, Simon J Day
}

\begin{abstract}
Ninety four neurologists in the United Kingdom, China, and West Germany responded to two structured questionnaires. The first assessed the diagnostic weighting assigned to a number of symptoms, signs, and clinical investigations ascertained from classical descriptions and case notes of patients with motor neuron disease (MND). The second tested the likelihood and consistency of diagnosis in a series of case summaries representing the clinical data of 10 patients with clinically and pathologically documented motor neuron disease. There was a wide measure of agreement concerning the common clinical features of the disease, especially regarding fasciculation of the tongue, fasciculation associated with weakness seen in more than one limb, and dysphagia. In the case summaries, however, there was clear variation in the ranked likelihood of the diagnosis of MND and in the consistency of diagnostic behaviour in the different groups of neurologists. These findings support the need for internationally agreed criteria in the diagnosis of MND. Any such criteria will need to be tested against a standardised data set to establish their validity.
\end{abstract}

Research on motor neuron disease (MND) depends on accurate diagnosis. At present there is no specific test for the disease, and diagnosis depends solely on clinical eriteria, based on positive features regarded as characteristic, especially a clinical syndrome with fasciculations of arms, legs, or tongue; muscular wasting and weakness; and a progressive course. The clinical features are usually consistent with one of the major clinical syndromes of MND, that is, amyotrophic lateral sclerosis, progressive muscular atrophy, or rarely, progressive spastic paraparesis. ${ }^{12}$ Other possible diagnoses, especially cervical and lumbar spondylosis with myelopathy, stroke, multiple sclerosis, spinal muscular atrophy, multiple mononeuropathy syndromes, polyneuropathy, and polymyositis must be excluded by appropriate investigation. ${ }^{1-4}$ In an analysis of the case records of 176 patients, 104 with MND and the remainder with cervical spondylosis, multiple sclerosis, or stroke, we devised a simple, fourstep algorithm for the diagnosis of MND that gave a $98 \%$ sensitivity and an $86 \%$ specificity when compared against another data set of 246 patients with MND, cervical spondylosis, multiple sclerosis, and stroke. ${ }^{5}$ This four step algorithm allows direct decision making based on the presence or absence of fasciculation in arms, legs, or tongue; muscular wasting in the limbs; sensory loss; and increased tendon reflexes. ${ }^{5}$

We studied the process of diagnosis of MND by neurologists in three different countries; firstly, by assessing the criteria currently used by these neurologists and, secondly, by scoring the ranking of the likelihood (probability) of diagnosis of MND and the consistency of their diagnoses in a standardised set of clinical case summaries taken from patients in whom this diagnosis had been established clinically and confirmed at necropsy examination.

\section{Material and methods}

Fifty British neurologists, 50 Chinese neurologists from the Beijing region, and 25 West German neurologists were invited to participate in the study. All the neurologists were fully trained and accredited in their own country, of consultant or equivalent grade, with hospital or university affiliations, and engaged in the full time practice of clinical neurology in a hospital or academic setting, or both.

\section{Study one: process of diagnosis of MND}

The first questionnaire listed a number of ' symptoms, signs and investigations, the presence or absence of which are generally recognised as relevant to the diagnosis of MND (see figure). ${ }^{1-3}$ Each neurologist was asked to rate the importance of the presence or absence of each symptom, sign, or result of clinical investigation in relation to its importance in making a diagnosis of MND by marking a $10 \mathrm{~cm}$ analogue scale for each item. On this scale $0 \mathrm{~cm}$ (left edge of line) ranked as zero weight for this diagnosis and $10 \mathrm{~cm}$ (right edge of line) ranked as maximum weight for the diagnosis. The data from this study were analysed by the method of repeated measurements analysis of variance. ${ }^{6}$ This statistical method addressed two questions; firstly, whether there was any difference in overall weight (importance) between the variables (that is, symptoms, signs, and investigations) and, secondly, whether there were differences in weight for any particular vari- 
able among the neurologists in the three countries. For this statistical analysis a logit transformation of the visual analogue scale measurements was made.

\section{Study two: likelihood and consistency of diagnosis of $M N D$}

In the second study the neurologists were presented with a set of 15 brief case summaries. These summaries consisted of 10 data sets of patients known from clinical and pathological evidence to have died from MND, obtained from the case records of the Royal London Hospital and the Radcliffe Infirmary, Oxford. Five of these data sets were given twice but disguised only by a change of sex and age (no less than one or more than five years). The data sets were reordered and presented on separate sheets; thus there were 15 apparently different case summaries. Each summary consisted of the clinical data including medical history and the results of investigations from onset to death, presented in conventional narrative form. The neurologists were asked to use a $10 \mathrm{~cm}$ visual analogue scale to mark the strength (relative likelihood) of a diagnosis of MND in each case. The left edge represented conviction that the diagnosis was not MND and the right edge certainty that the diagnosis was MND. No indication was given in the proforma on the diagnosis or even as to whether all or only some of the summaries represented patients with MND.

The data from study 2 were analysed with respect to likelihood and consistency of diagnosis from the analogue score measurements. In the likelihood analysis a score of 5 or more was considered as "likely to be MND" and a score of less than 5 as "unlikely to be MND". We used the terms "likely" and "unlikely" because they most closely resemble the usual process of clinical diagnosis in which a diagnosis is simply regarded as likely or unlikely and different items of information are used in a relatively non-probabalistic way in achieving a working diagnosis (see discussion). Consistency of diagnosis was assessed by considering how respondents classified each pair of duplicate case summaries. For each pair of duplicated cases a two-way table was constructed with four cells; one in which both cases were classified as "likely to be MND", one in which both were classified as "unlikely to be MND", one in which the first was "likely" and the second "unlikely", and one in which the first was "unlikely" and the second "likely". In this way the binary observations were standardised so that we could assess the proportion of observers who made the same observation twice (consistent responders) and those who did not.

\section{Results}

Responses were obtained from 39 of 50 British neurologists, 40 of 50 Chinese neurologists, and 15 of 25 West German neurologists, representing a $75 \%$ overall response rate.
Study 1: process of diagnosis of MND

The neurologists in all three countries consistently rated motor symptoms, especially fasciculation, weakness, dysphagia, and dysarthia, as important in diagnosing $\mathrm{MND}$, but there were differences in emphasis of certain symptoms. British and West German neurologists assigned less importance to difficulty in writing, rapid fatigue, and muscle spasm than Chinese neurologists. There was a clear recognition ( $p=0.0001)$ of the importance of these motor symptoms compared with incontinence, seizures, diplopia, sensory loss, and visual loss, all of which were regarded as inconsistent with a diagnosis of MND (figure), although Chinese neurologists placed less weight on this than their European colleagues $(p=0.0001)$. Absence of rapid fatigue and, to a lesser extent, absence of muscle cramps, muscle spasm, and fasciculations in more than one limb, were regarded by British neurologists as making the diagnosis of MND unlikely, but the Chinese and West German neurologists placed less negative emphasis on the absence of these features.

Physical signs, graded in rank order in relation to the diagnosis of $M N D$, ranged from fasciculation of the tongue and of muscles in more than one limb, representing signs with high ranking for the diagnosis of MND, to sensory loss, a sign ranking very low in relation to a diagnosis of MND. Other characteristic features of lower motor neuron lesions and signs of upper motor neuron lesion were ranked as less important than fasciculation, although there was some disagreement among the three groups, particularly with regard to spasticity which was rated much more characteristic of MND by British and West German neurologists than by Chinese neurologists. Absence of these physical signs was assessed with similar emphasis as moderately inconsistent with the diagnosis of MND (scores ranging from 3 to 5) by all three groups of neurologists.

The assessment of the results of clinical investigations showed that the presence of neurogenic change in a muscle biopsy specimen was universally regarded as an important positive feature, and the presence of cervical spinal canal stenosis as an important negative feature, while all the other investigations were generally regarded as less relevant $(p=0.0001)$. There were, however, clear differences in the ratings ascribed to these other investigations, particularly with regard to cervical and lumbar spondylosis and a normal EMG, which were rated as more important negative features in China than in Britain or West Germany. A raised CSF protein conveyed more negative bias than a normal $\mathrm{CSF}$ protein, and a slightly raised blood CK level (195 IU/1) was rated as of intermediate diagnostic importance.

\section{Study 2: likelihood and consistency of diagnosis} of $M N D$

There was a reasonable level of diagnostic agreement between the neurologists in the three countries in all except four cases. Two of 

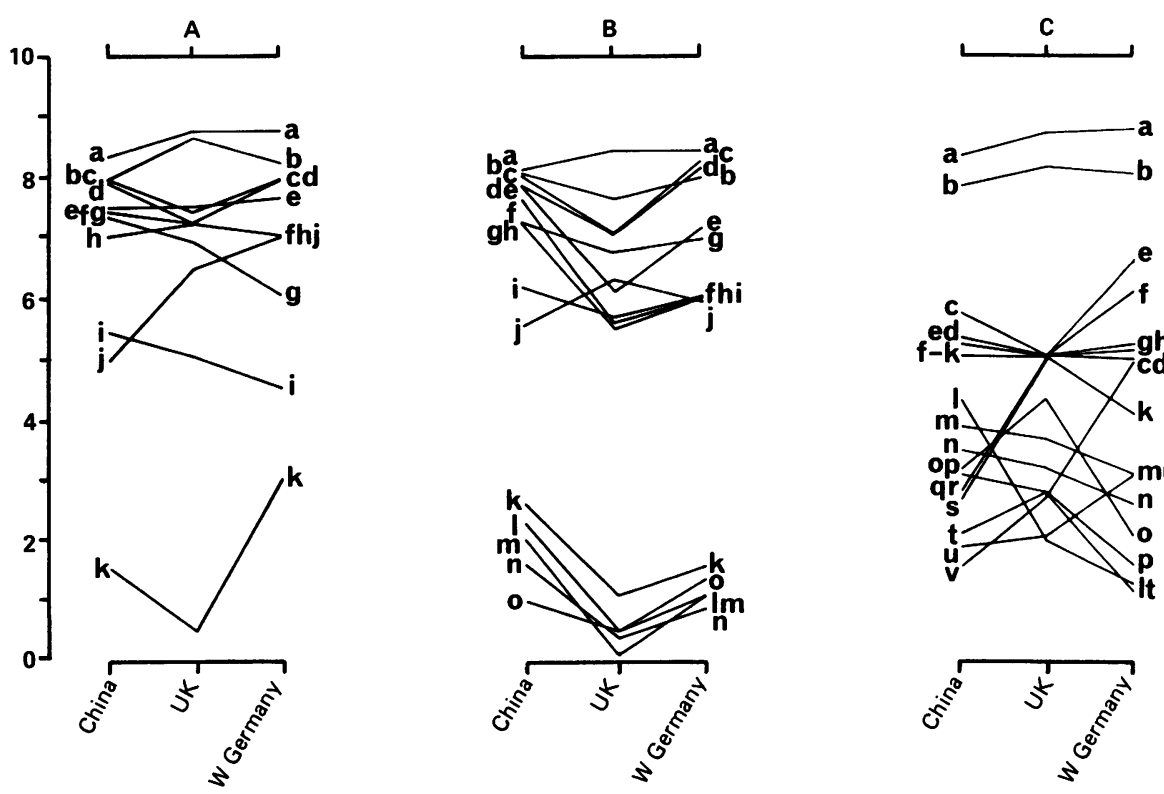

Figure Study 1: mean ranks ascribed to various physical signs $(A)$, symptoms (B), and results of clinical investigations $(C)$ in diagnosis of motor neuron disease (MND). Ordinate represents rankings marked by respondent neurologists from three countries on $10 \mathrm{~cm}$ analogue scale, where $10 \mathrm{~cm}$ represented maximum weight and $0 \mathrm{~cm}$ minimum weight for diagnosis of $M N D$.

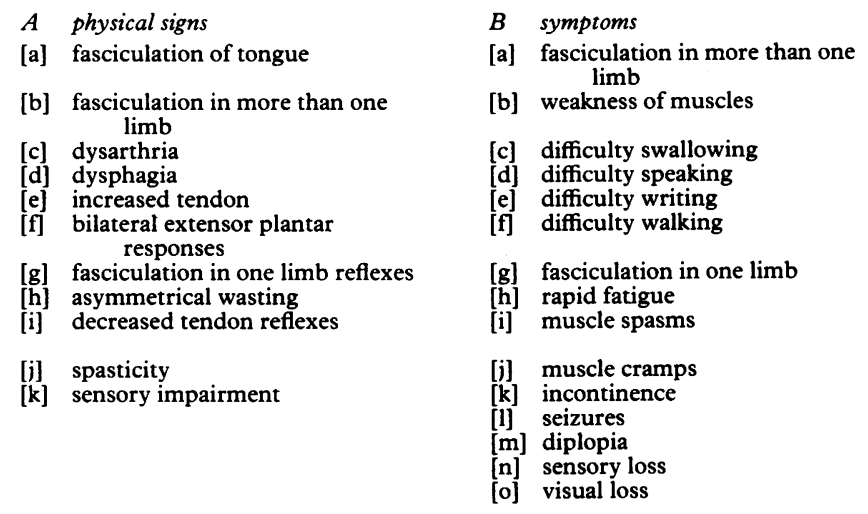

C results of clinical investigations

[a] muscle biopsy specimen; neurogenic change

[b] myelogram; cervical canal stenosis

[c] $x$ ray; lumbar spine normal

[d] $x$ ray; cervical spine normal

[e] thyroid function

[g] creatine kinase $195 \mathrm{IU} / 1$

[h] CT brain; normal

[i] EMG; widespread neurogenic change

[j] thyroid function abnormal

[k] creatine kinase normal

1] muscle biopsy; normal

[m] CSF protein $100 \mathrm{mg} / \mathrm{l}$

[n] myelogram normal

[o] diabetes mellitus

p] CSF serology + ve

q] ray; cervical spondylosis

r] $x$ ray; lumbo-sacral spondylosis

[s] EMG; normal

[t] Serum $B_{12} 170 \mathrm{pg} / \mathrm{ml}$

[v] CT brain; cerebrovascular disease

these four cases consisted of a duplicated pair of summaries. One case occupied an equivocal position. There was consistency among the respondents in relation to two duplicated cases in which the clinical syndrome of MND was fully expressed, as recognised in standard descriptions of the clinical features of the disease. ${ }^{1-3}$ In three pairs of duplicated cases in which the clinical syndrome was less fully developed, however, differences in diagnostic behaviour among the neurologists in the three countries were distinct. In four of the 10 unduplicated cases there was a consensus about the unlikelihood of a diagnosis of MND. For each of the five pairs of duplicated cases the degree of consistency in diagnosis was $96 \%$, $94 \%, 77 \%, 72 \%$, and $65 \%$.

\section{Discussion}

These two investigations were designed to assess the importance ascribed to various common symptoms, signs, and clinical investiga- tions in establishing a diagnosis of MND by neurologists in Britain, West Germany, and China (study 1) and, in a series of case summaries of patients known to have MND, to assess the probability and consistency of the diagnostic process (study 2). The neurologists in the three countries were all of equivalent status in relation to their affiliations, appointments, and experience. In study 1 most of the neurologists agreed on the importance of fasciculation of the tongue, fasciculation in more than one limb, weakness, dysphagia, and dysarthria in the diagnostic process. ${ }^{1-3}$ Less specific clinical features-for example, the presence of upper motor neuron signs-were accorded less weight. Clinical investigation seemed to be assessed critically-for example, neurogenic change in a muscle biopsy specimen and the presence of canal stenosis in radiographs of the cervical or lumbar spine were regarded as relevant positive and negative features respectively. A raised CSF protein provoked suspicion of another diagnosis, and a mildly raised 
blood creatine kinase level was regarded as consistent with the diagnosis but not as a feature of major diagnostic importance. Other features such as cervical spondylosis were rated as important negative features in Britain and Germany, but seemed to have a more negative emphasis in China. Many of the investigations commonly employed achieved rankings suggesting that the information they revealed contributed neither positively nor negatively to the diagnosis.

In study 2 there were 10 case summaries comprising known cases of MND, with five duplicate cases disguised only by a change of sex and age. There were clear differences in rating of diagnostic likelihood between the neurologists in the three countries, including both unduplicated and duplicated cases. Thus there was lack of agreement between neurologists in different countries in the same cases, and in three cases of MND the diagnosis was regarded as unlikely. In study 2 the neurologists were not given any indication as to study design and may well have been expecting some of the patients to have some other neurological diagnosis, an expectation which resembles the reality of clinical practice. There was consistency among the respondents in relation to two duplicated cases in which the clinical syndrome of MND was fully expressed, as recognised in standard descriptions of the clinical features of the disease. ${ }^{1-3}$ On the other hand, in three pairs of duplicated cases in which the clinical syndrome was less fully developed differences in diagnostic behaviour among the neurologists in the three countries were distinct.

The disparity between the wide measure of agreement in relation to the clinical features of the disease that have diagnostic importance and the results of the analysis of probability of diagnosis and of consistency of diagnostic behaviour in the case summaries, suggest that the respondent neurologists agreed with each other in theoretical terms, but that they acted differently when asked to make diagnostic judgements. That is, they utilised their diagnostic database in different ways. This is particularly shown by the inconsistent diagnostic responses given in study 2 when there was conflict within the clinical data. Although this analysis of diagnostic behaviour in paper cases might be considered a theoretical exercise, Kirwan et al showed that assessments based on case summaries correlate highly $(r=+0.9)$ with those made on the equivalent real patients. ${ }^{7}$ In real life clinical practice, when confronted with a potentially serious diagnosis such as MND with its implications of untreatability and early death, most neurologists actively consider alternative diagnoses in order not to miss a treatable condition. In this situation therefore the neurologist might be expected to adopt a strategy of differential diagnosis rather than of a single diagnosis. In study 2 , however, the degree of inconsistency in diagnostic behaviour between cases was distinct, and even extended across matching pairs of cases. There seems to have been conflict in the minds of the respondent neurologists between a desire to make a unitary diagnosis and an attempt to keep diagnostic options open by allowing uncertainty. The reasoning process utilised in clinical diagnosis are complex and poorly understood. Kassirer concluded that probabilistic reasoning, using statistical relationships between clinical findings and diagnosis, causal reasoning, in which the clinical findings are assessed for coherency in relation to a physiological model of the disease, and deterministic reasoning, in which a set of compiled rules, based on previous experience is followed, are all utilised in the process of diagnosis in clinical practice. ${ }^{8}$ These methods are probably complementary, and current understanding of their application and of their interrelationships is imprecise. In clinical practice probabilistic methods are probably little used. ${ }^{8}$ In study 2 we used the rating of diagnostic strength as a means of assigning the likelihood or unlikelihood of the diagnosis of MND. This interpretation supposes that the neurologists would assume the diagnosis to be likely or unlikely and that they would not take up an intermediate position. This accords with the use of either a non-probabilistic or deterministic pattern of diagnostic behaviour. In the latter, however, different weights might have been applied to certain items of information. Our study did not address this problem, and we therefore preferred not to apply any arbitrary sub-divisions to the scores given by the marks placed on the $10 \mathrm{~cm}$ scales.

Our results illustrate the need, not only to define clearly the criteria used for the diagnosis of MND but also to understand more completely the ways in which these criteria are used in different diagnostic situations. For example, in clinical situations in which the data-base is incomplete, perhaps because the clinical syndrome is incompletely developed or because there are unusual features. The inherent conflict in the data seems to be dealt with by different neurologists in different ways, leading to apparently different diagnostic behaviours. Any agreed set of criteria for diagnosis of MND, as currently under consideration by the World Federation of Neurology, will need to be validated prospectively. These criteria could be tested against a data-set of case summaries with a similar analytical design to the one we have employed.

This work was supported by the Wellcome Trust, and the Motor Neurone Disease Association. We thank the 85 neurologists in Britain, West Germany, and China who responded to ou questionnaires. Dr Mark Chaput de Saintonge and Professo W B Matthews gave helpful advice in planning the two studies. Full details of the numerical results are available on request from MS.

1 Walton JN. Brain's diseases of the nervous system. 9th ed. Oxford: Oxford University Press, 1985.

2 Rowland LP. Diverse forms of motor neuron disease. In: Rowland LP, ed. Human motor neuron diseases. New York: Raven Press, 1982:1-13.

3 Swash M, Schwartz MS. Neuromuscular diseases; a practical approach to diagnosis and management. London: Springerapproach to diagn
Verlag, 1988.

4 Mitsumoto $\mathrm{H}$, Hanson MR, Chad DA. Amyotrophic lateral sclerosis. Arch Neurol 1988;45:189-202.

5 Li T-M, Day SJ, Alberman E, Swash M. Differential diagnosis of motor neuron disease from other neurological conditions, Lancet 1986;2:731-3.

6 Hand DJ, Taylor CC. Multivariate analysis of variance and repeated measures; a practical approach for behavioural scientists. London: Chapman Hall, 1987.

7 Kirwan JR, Chaput de Saintonge DM, Joyce CRB, Currey HLF. Clinical judgement in rheumatoid arthritis. In: Rheumatologists' diagnoses and the development of "paper patients". Ann Rheumatic Dis 1983;42:644-7.

8 Kassirer JP. Diagnostic reasoning. Ann Intern Med 1989; 110:893-900. 